\title{
Cervical surveillance in HIV-positive women: a genitourinary medicine clinic experience
}

\author{
Fatima W Ibrahim, Gabriel Schembri, Huda Taha, Sati Ariyanayagam, Jyoti Dhar
}

\begin{abstract}
Background The prevalence of cervical intraepithelial neoplasia $(\mathrm{CIN})$ is increased in HIV infection. The UK National Health Service Cervical Screening Programme (NHSCSP) guidelines therefore provide specific recommendations for HIV-positive women. An audit of cervical surveillance in HIV-positive women who attend the genitourinary medicine (GUM) department at the Leicester Royal Infirmary, Leicester, UK was conducted. The objectives were to assess adherence to UK and local screening guidelines, prevalence of cervical pathology and appropriate referral for colposcopy.
\end{abstract}

Methods A retrospective case note review of 130 HIVinfected women attending the GUM department between January 2000 and December 2005 was undertaken.

Results Results showed that $76.2 \%$ of patients had cervical cytology within a year of HIV diagnosis and $42.4 \%$ of patients had abnormal cytology. All patients with dyskaryosis were referred for colposcopy according to local and national guidelines. Cytology results were

\section{Introduction}

The prevalence of cervical intraepithelial neoplasia (CIN) is increased in HIV infection ${ }^{1}$ and invasive cervical cancer is classed as an AIDS-defining illness by the United States Centers for Disease Control and Prevention. Human papillomavirus (HPV) has been strongly implicated in the pathogenesis of CIN and cervical cancer. HIV-positive women have a higher prevalence of cervical HPV infection and are more likely to have persistent HPV infection than HIV-negative women. ${ }^{2}$ Treatment with highly active antiretroviral therapy (HAART) has not been shown to significantly clear cervical HPV infection or induce regression of CIN. ${ }^{2}$ It is therefore very important to regularly screen for and treat CIN in all HIV-positive women, including those who have responded to treatment with HAART. Annual cytology is therefore recommended in HIV-positive women, and not 3-yearly as in HIVnegative women, with an initial colposcopy where resources are available (NHSCSP guidelines, 2004). ${ }^{3}$ Many genitourinary medicine (GUM) departments in the UK are responsible for ensuring that cervical surveillance is

University Hospitals of Leicester NHS Trust, Department of Genitourinary Medicine, Leicester Royal Infirmary, Leicester, UK Fatima W Ibrahim, MBChB, DTM\&H, Specialist Registrar

Gabriel Schembri, MRCP, Specialist Registrar

Jyoti Dhar, MRCP, DTM\&H, Consultant

Department of Genitourinary Medicine, Chesterfield Royal Hospital, Calow, UK

Huda Taha, MBBs, Staff Grade Doctor

Peterborough and Stamford Hospitals NHS Foundation Trust, Department of Sexual Health, Edith Cavell Hospital,

Peterborough, UK

Sati Ariyanayagam, FRCOG, FFSRH, Consultant

Correspondence to: Dr Fatima W Ibrahim, University Hospitals of Leicester NHS Trust, Department of Genitourinary Medicine, Leicester Royal Infirmary, Infirmary Square,

Leicester LE1 5WW, UK. E-mail: drfwih70@yahoo.com consistent with histological findings and the prevalence of CIN was $15.2 \%$. CD4 counts at presentation were significantly lower in those with dyskaryosis compared with those without dyskaryosis $(p=0.038)$. Twenty-two patients were lost to follow-up after initial cytology.

Discussion and conclusions $A$ designated health advisor in the GUM department co-ordinates cervical surveillance in HIV-positive women. This, together with an increasingly integrated service with family planning services, may contribute to relatively successful surveillance. Overall, patients are carefully monitored to ensure that surveillance is adequate. Extra vigilance is, however, required and further cost-effective measures in future may include more active involvement of general practitioners.

Keywords audit, cervical surveillance, genitourinary medicine, HIV, integrated services

J Fam Plann Reprod Health Care 2009; 35(2): 101-103

(Accepted 30 September 2008)

\section{Key message points}

- Effective measures are required to ensure that cervical surveillance is adequate in HIV-infected women as they are at higher risk of developing cervical intraepithelial neoplasia.

- A designated health advisor or similarly trained professional who co-ordinates screening and integrated services with family planning may aid successful surveillance.

- Future measures for effective screening may include more active involvement of general practitioners.

adequate as they also monitor the HIV infection. In our department cytology is offered to all newly diagnosed HIVpositive women. Subsequent surveillance interval and referral for colposcopy are determined by results and local and national guidelines. According to our local guidelines, at initial HIV diagnosis women are not referred for colposcopy if cytology is negative, women with moderate and severe dyskaryosis are referred for colposcopy, women with borderline or mild changes have cytology repeated after a 6-month interval and a referral for colposcopy made if the abnormality has persisted or progressed. Women with negative cytology have annual smears. A designated health advisor co-ordinates surveillance. This process is aided by an increasingly integrated service with the family planning clinic, so that HIV-infected women who attend for contraceptive advice are also offered sexually transmitted infection (STI) screening and cervical cytology accordingly.

We conducted an audit of cervical surveillance in HIVpositive women attending our GUM department to assess adherence to recommended UK NHSCSP and local guidelines.

\section{Methods}

A retrospective case note review of 130 HIV-infected women attending the GUM department between January 2000 and December 2005 was undertaken. A total of 101 cases were audited as 21 patients were lost to follow-up 
Table 1 Social and demographic characteristics of the cohort patients $(n=101)$

\begin{tabular}{lrr}
\hline Characteristic & $\boldsymbol{n}(\%)$ & \\
\hline Age (years) & & \\
$<16$ & 0 & $(0)$ \\
$16-19$ & 1 & $(1.0)$ \\
$20-24$ & 10 & $(9.9)$ \\
$25-34$ & 61 & $(60.4)$ \\
$35-44$ & 24 & $(23.7)$ \\
$45+$ & 5 & $(5.0)$ \\
Ethnicity & & \\
$\quad$ Black African & 21 & $(90.1)$ \\
Caucasian & 4 & $(2.0)$ \\
Indian & 2 & $(2.0)$ \\
Other & 2 & $(2.0)$ \\
Not recorded & & \\
Asylum seeker & $24 / 56$ & $(42.9)$ \\
Yes & $32 / 56$ & $(57.1)$ \\
No & 45 & \\
Not recorded & & \\
\hline
\end{tabular}

Table 2 Results of initial cytology, methods of contraception and treatment with highly active antiretroviral therapy (HAART) $(n=99)$

\begin{tabular}{lrr}
\hline Characteristic & $\boldsymbol{n}(\%)$ & \\
\hline Cytology result & & \\
Negative & 57 & $(57.6)$ \\
Borderline & 14 & $(14.1)$ \\
Dyskaryosis: & 28 & $(28.3)$ \\
Mild & $16 / 28$ & $(57.1)$ \\
$\quad$ Moderate & $8 / 28$ & $(28.6)$ \\
$\quad$ Severe & $4 / 28$ & $(14.3)$ \\
Contraception & 7 & $(7.1)$ \\
$\quad$ Barrier & 23 & $(23.2)$ \\
Hormonal & 2 & $(2.0)$ \\
Intrauterine device & 2 & $(2.0)$ \\
Sterilised & 55 & $(55.6)$ \\
None & 10 & $(10.1)$ \\
Not recorded & & \\
HAART & 40 & $(40.4)$ \\
Yes & 59 & $(59.6)$ \\
No & & \\
\hline
\end{tabular}

before cytology, seven were pregnant at the time of evaluation and due for cytology after delivery and one patient had previously had a total abdominal hysterectomy (TAH). Data were collected for patients' age, ethnic origin, immigration status, treatment with HAART, methods of contraception and history of other STIs. Cytology and histology results were recorded. The Statistical Package for the Social Sciences (SPSS) (Chicago, IL, USA) was used for statistical analysis. The Mann-Whitney test was used to compare CD4 counts between the normal and dyskaryotic groups. Categorical data were analysed using the Chisquare test.

\section{Results}

In our cohort, 99/101 women had cytology performed within a year of diagnosis of HIV infection. Only two women had no documented evidence of cytology being offered. A total of 22 women were lost to follow-up after this initial cytology.

Table 1 shows the social and demographic characteristics of the cohort. The mean age was 32 years (standard error 0.63 ) and 91/101 $(90.1 \%$ ) of the women were of Black African origin. Immigration status was documented for 56 women and, of those, 24 (42.9\%) were asylum seekers.

Table 2 shows cytology results, methods of contraception and treatment with HAART. Of 99 women, $57(57.6 \%)$ had negative cytology, $14(14.1 \%)$ had borderline change and $28(28.3 \%)$ had mild, moderate or severe dyskaryosis at presentation. Fifty-five (55.6\%) women used no contraception. When contraception was used, hormonal methods were preferred. Forty (40.4\%) women were on HAART.

CD4 counts at presentation were significantly lower in the dyskaryotic group [median 220, interquartile range (IQR) 130-340] than in the non-dyskaryotic group (median 370 , IQR 180-520; $p=0.038$ ). Comparisons of categorical data between dyskaryotic and non-dyskaryotic patients on HAART, using contraception and with a history of concurrent or previous STIs were not statistically significant ( $p=0.08,0.92$ and 0.59 , respectively).

\section{Colposcopy and follow-up}

Follow-up cytology and colposcopy results are shown in Table 3. Following the initial smear, all women were appropriately followed up with annual cytology or referred for colposcopy according to local and national guidelines. In the borderline group, nine women had normal cytology, three had persistent borderline change and were due for referral for colposcopy, one developed moderate dyskaryosis (CIN2 on histology) and one was lost to follow-up.

In the mild dyskaryosis group, two patients had normal cytology, three with persistent mild dyskaryosis had normal histology results following colposcopy, one result was awaited, one woman developed moderate dyskaryosis (CIN3 on histology) and two developed severe dyskaryosis (CIN1 and CIN3 on histology). Three women were pregnant and due for further cytology after delivery, two women were due to have further cytology and two women were lost to follow-up.

In the moderate dyskaryosis group, six out of eight women had CIN2 following colposcopy and histology, one had CIN3 and one patient was lost to follow-up. All four patients with severe dyskaryosis were found to have CIN3. There was a default rate of $50 \%$ for attendance for the initial colposcopy appointment; however, all but one patient subsequently attended following recall.

Table 3 Follow-up cytology and colposcopy

\begin{tabular}{|c|c|c|c|c|c|}
\hline \multirow[t]{2}{*}{ Initial cytology } & \multicolumn{5}{|c|}{ Follow-up cytology and histologya } \\
\hline & Negative & Borderline & $\begin{array}{l}\text { Dyskaryosis } \\
\text { mild }\end{array}$ & $\begin{array}{l}\text { Dyskaryosis } \\
\text { moderate }\end{array}$ & $\begin{array}{l}\text { Dyskaryosis } \\
\text { severe }\end{array}$ \\
\hline Borderline $(n=14)$ & 9 & 3 & & 1 (CIN2) & \\
\hline Dyskaryosis: mild ( $n=16)$ & 2 & & $\begin{array}{l}4 \text { (3 normal, } \\
1 \text { awaited) }\end{array}$ & $1(\mathrm{CIN} 3)$ & $2(1 \mathrm{CIN} 1,1 \mathrm{CIN} 3)$ \\
\hline Dyskaryosis: moderate $(n=8)$ & & & & $\begin{array}{l}6 \text { (CIN2) } \\
1 \text { (CIN3) }\end{array}$ & \\
\hline Dyskaryosis: severe $(n=4)$ & & & & 4 (CIN3) & \\
\hline
\end{tabular}

aFour patients lost to follow-up, three pregnant and two due for cytology.

$\mathrm{CIN}$, cervical intraepithelial neoplasia. 


\section{Discussion}

This audit showed that HIV-infected women followed up in our GUM department are being offered cervical cytology and are referred for colposcopy appropriately in accordance with UK NHSCSP and local screening guidelines. Some $76.2 \%$ of all the $130 \mathrm{HIV}$-positive female patients eligible for screening were offered cervical smears according to the current recommendations, as compared to rates of $50.3 \%$ and $54 \%$ quoted in other audits. ${ }^{4,5}$ Where smears were not performed, this was generally in patients who defaulted despite repeated recall or because cytology was not indicated at the time (this was due to pregnancy in seven patients and a TAH in one patient). This relative success may have been possible through the designation of a health advisor who co-ordinates cervical cytology in HIVinfected women. Further studies are, however, needed to assess this. A database of these cytology details is kept within the GUM department and an in-house call and recall system operated with patients being written to and/or telephoned when cytology is due. Services are increasingly being integrated with family planning and this may have aided uptake and surveillance as every opportunity is taken to ask about smear tests and whether these are up to date. Such integrated services have been shown to improve sexual and reproductive service provision in HIV-positive women. ${ }^{6}$

Extra vigilance has been required as most of our patients belong to ethnic minority and refugee groups and low coverage has been documented among this group of women, who are also a mobile population ( 22 patients in our audit were lost to follow-up after initial screening)..$^{7-9}$ They may have originated from countries where there is no screening programme and therefore do not realise the importance of screening. Other (mainly social) issues, such as pending immigration cases in asylum seekers, may take priority over attending for clinic appointments. Another reason given for defaulting is the fear of being informed of yet another condition in addition to HIV infection (i.e. cervical cancer). We have tried to overcome such potential barriers for successful cervical surveillance by providing support, careful explanation, understanding cultural barriers and dispelling any myths and misconceptions about the programme. The regular attendance by our patients for the management of HIV infection, for monitoring purposes and medication, has aided successful screening as every opportunity is taken to provide further education and to ensure that cytology is up to date. We could, however, improve our surveillance of patients further by offering cytology earlier after the diagnosis of HIV infection is made and before patients are lost to follow-up.

There are potential cost implications associated with the time spent calling and recalling patients who have defaulted. With improved long-term survival in HIV infection, further cost-effective measures may have to be developed to maintain effective cervical surveillance. This may include shifting some of the responsibility on to general practitioners (GPs). However GPs should be made aware that HIV-positive women require annual and not 3yearly screening.

\section{Conclusions}

The results in our cohort of patients showed an association between lower CD4 counts and cervical disease, and therefore the importance of early screening. This audit showed that HIV-infected women attending the GUM department at the Leicester Royal Infirmary are being carefully monitored to ensure that cervical surveillance is carried out according to UK NHSCSP and local guidelines. Despite this close monitoring and an established call and recall system, there was a high default rate following initial cytology. Further cost-effective measures are therefore needed to provide extra vigilance in order to avoid further morbidity and mortality in these HIV-infected women. This may include offering cytology sooner after HIV diagnosis, and more active involvement of GPs.

\section{Statements on funding and competing interests \\ Funding None identified.}

Competing interests None identified.

\section{References}

1 Six C, Heard I, Bergeron C, Orth G, Poveda JD, Zagury P, et al. Comparative prevalence, incidence and short-term prognosis of cervical squamous intra-epithelial lesions among HIV-positive and HIV-negative women. AIDS 1998; 12: 1047-1056.

2 Palefsky JM. Cervical human papillomavirus infection and cervical intraepithelial neoplasia in women positive for human immunodeficiency virus in the era of highly active antiretroviral therapy. Curr Opin Oncol 2003; 15: 382-388.

3 Colposcopy and Programme Management: Guidelines for the NHS Cervical Screening Programme (NHSCSP Publication No. 20). April 2004. http://www.cancerscreening.nhs.uk/ cervical/publications/nhscsp20.pdf [Accessed 26 June 2008].

4 Vonau BU, Boag F, Barton SE. Audit of cervical screening and colposcopy in HIV positive women at a central London Teaching Hospital. Int J STD AIDS 1999; 10: 755-758.

5 Morris EJ, Bates S. Audit of cervical cytology in HIV positive women. Int J STD AIDS 2007; 18: 126-127.

6 Coyne KM, Hawkins F, Desmond N. Sexual and reproductive health in HIV-positive women: a dedicated clinic improves service. Int J STD AIDS 2007; 18: 420-421.

7 Fylan F. Screening for cervical cancer: a review of women's attitudes, knowledge and behaviour. Br J Gen Pract 1998; 48: 1509-1514.

8 Kernohan EEM. Evaluation of a pilot study for breast and cervical cancer screening with Bradford's minority ethnic women; a community development approach 1991-1993. Br J Cancer 1996; 74(Suppl. XXIX): S42-S46.

9 Schwartz M, Savage W, George J, Emohare L Women's knowledge and experience of cervical screening: a failure of health education and medical organization. Community Med 1989; 11: 279-289.

\section{PEER REVIEWERS/JOURNAL REVIEW WRITERS WANTED}

\section{PEER REVIEWERS}

If you have a special interest in one or more of the topics covered by the Journal and have some time available to peer review occasional papers in your own area(s) of expertise then perhaps you might be interested in joining the Journal's team of peer reviewers? In common with the majority of other academic journals, peer reviewers offer their services on a voluntary basis; however, if you are a member of the Faculty of Sexual and Reproductive Healthcare then each completed review counts for two (2) CME points.

\section{JOURNAL REVIEW WRITERS}

The Journal regularly reviews articles from related publications (see page 124) and is looking to recruit more volunteers to compile these short reviews. For further information and/or to offer your services (at most once or twice a year) please email your contact details to the Journal Editorial Office together with a note of any special interests and/or expertise to assist us in allocating relevant articles to individual reviewers.

For further information on either of these roles please contact the Journal Editorial Office at journal@fsrh.org. 